\title{
Reduced interactivity during microbial community degradation lead to the extinction of Tricholomas matsutake
}

\author{
Hanchang Zhou ${ }^{1}$, Anzhou Ma ${ }^{2}$, Liu Guohua ${ }^{1}$, Xiaorong Zhou ${ }^{1}$, Jun Yin ${ }^{1}$, Yu Liang ${ }^{1}$, Feng \\ Wang $^{3}$, and Guoqiang Zhuang ${ }^{1}$ \\ ${ }^{1}$ RCEES \\ ${ }^{2}$ Research Centre for Eco-Environmental Sciences Chinese Academy of Sciences \\ ${ }^{3}$ Jiangsu University
}

June 9, 2021

\begin{abstract}
Ecosystem degradation is a process during which different ecosystem components interact and affect each other. The microbial community, as a component of the ecosystem whose members often display high reproduction rates, is more readily able to respond to environmental stress at the compositional and functional levels, thus potentially threatening other ecosystem components. However, very little research has been carried out on how microbial community degradation affects other ecosystem components, which hampers the comprehensive understanding of ecosystems as a whole. In this study, we investigated the variation in a soil microbial community through the extinction gradient of an ectomycorrhizal species (Tricholomas matsutake) and explored the relationship between microbial community degradation and ectomycorrhizal species extinction. The result showed that during degradation, the microbial community switched from an interactive state to a stress tolerance state, during which the interactivity of the microbial community decreased, and the reduced community interactions with T.matsutake marginalized it from a large central interactive module to a small peripheral module, eventually leading to its extinction. This study highlights the mechanisms of T.matsutake extinction due to the loss of soil microbial community interactivity, offering valuable information about soil microbial community degradation and the plant ectomycorrhizal species conservation.
\end{abstract}

Reduced interactivity during microbial community degradation lead to the extinction of Tricholomas matsutake

\section{Authors}

Hanchang Zhou ${ }^{\text {a, b }}$, Anzhou Ma ${ }^{\text {a, b, }}{ }^{*}$, Guohua Liu ${ }^{\text {a, b }}$, Xiaorong Zhou ${ }^{\text {a, b }}$, Jun Yin ${ }^{\text {a, b }}$, Yu Lianga, b, Feng Wang ${ }^{\mathrm{c}}$, Guoqiang Zhuanga, b, ${ }^{*}$

${ }^{a}$ Research Center for Eco-Environmental Sciences, Chinese Academy of Sciences, 100085 Beijing, China

b University of Chinese Academy of Sciences, 100049 Beijing, China

${ }^{c}$ School of Food and Biological Engineering, Jiangsu University, 212013 Zhenjiang, China

\section{*Corresponding author:}

Anzhou Ma

Phone: 86-10-6284-9156, Fax No.: 86-10-6284-9156

Email: azma@rcees.ac.cn

Guoqiang Zhuang 
Phone: 86-10-6284-9613, Fax No.: 86-10-6284-9613

Email: gqzhuang@rcees.ac.cn

\begin{abstract}
Ecosystem degradation is a process during which different ecosystem components interact and affect each other. The microbial community, as a component of the ecosystem whose members often display high reproduction rates, is more readily able to respond to environmental stress at the compositional and functional levels, thus potentially threatening other ecosystem components. However, very little research has been carried out on how microbial community degradation affects other ecosystem components, which hampers the comprehensive understanding of ecosystems as a whole. In this study, we investigated the variation in a soil microbial community through the extinction gradient of an ectomycorrhizal species (Tricholomas matsutake ) and explored the relationship between microbial community degradation and ectomycorrhizal species extinction. The result showed that during degradation, the microbial community switched from an interactive state to a stress tolerance state, during which the interactivity of the microbial community decreased, and the reduced community interactions with T.matsutake marginalized it from a large central interactive module to a small peripheral module, eventually leading to its extinction. This study highlights the mechanisms of T.matsutakeextinction due to the loss of soil microbial community interactivity, offering valuable information about soil microbial community degradation and the plant ectomycorrhizal species conservation.
\end{abstract}

Key words: microbial community, degradation, network, interactivity, Tricholomas matsutake

\title{
Introduction
}

Environmental stresses due to climate change and human activity are threatening to degrade the biodiversity and services of terrestrial ecosystems around the world (Singh et al. , 2010, De \& Ashley, 2013, Jansson $\&$ Hofmockel, 2020). An ecosystem contains a myriad of components that interact and affect each other in complex ways. Thus, the degradation of a few, or even just one component, could potentially change the state of others and lead to holistic ecosystem degradation (Moreno-Mateos et al. , 2020, Cheng et al. , 2021). Among ecosystem components, the microbial community is unique. On the one hand, its members have extremely high richness and reproduction rate, which allows the microbial community to be responsive to stress as its composition can adapt rapidly when facing environment fluctuations (Jansson \& Hofmockel, 2020, Hall et al. , 2018). On the other hand, the microbial community plays an irreplaceable role in decomposition, nutrient cycling, and regulation of above-ground community diversity and activity, therefore, degradation of the microbial community is a potential threat to related ecosystem functions and other ecosystem components (Berendsen et al. , 2012).With rising awareness of the importance of the microbial community and increasingly mature methodology, research on ecosystem degradation related to microbial communities under climatic and anthropogenic stress have begun to receive increasing attention (Firestone, 2006, Hallet al. , 2018).

However, most microbial community degradation related research has been performed according to vegetation or soil degradation gradients, rather than according to microbial community degradation itself (Zhang et al. , 2010, Cheng et al. , 2021). These studies have also mainly focused on the effects that vegetation or soil degradation exert on the microbial community, either directly or indirectly, rather than the effects that microbial community degradation exerts on other ecosystem components (Zhang et al. , 2010, Cheng et al. , 2021). Yet, due to the much higher reproduction rate of microbes than plants, the microbial community could have already passed through numerous generations and established new stages of succession before significant degradation of vegetation or soil is detected (Curtis, 2006, Konopka, 2006, Berendsen et al. , 2012). Thus, it is more plausible that microbial community degradation occurs earlier than vegetation degradation, and that the latter is a consequence of microbial community degradation (Curtis, 2006, Konopka, 2006, Berendsen et al., 2012). However, studies exploring the effects of microbial community degradation on other ecosystem components, such as ectomycorrhizal species, remain scarce.

Ectomycorrhizal fungi are fungal microbes that live in the interface between plant roots and soils, and are 
possibly the first step that soil microbial community degradation exert to plant community (Martin et al., 2016). The cloze interactions between ectomycorrhizal fungi species and host plants had been comprehensively studied from the molecular level to ecological level (Martin et al., 2016, Hoeksema et al., 2018). However, the interactions between ectomycorrhizal fungi species and soil microbial community were seldom reported. Theoretically, since the ectomycorrhizal fungi physically interact with the soil microbial community, the ectomycorrhizal fungi species should also interact at the biochemical or ecological level (Bowen \& Theodorou, 1979). In recent years, climate change has resulted in the decreasing production and the regional extinction of ectomycorrhizal fungi species in several ecosystems even if the vegetation has not yet shown significant degradation (Collado et al. , 2019, Ohara \& Hamada, 1967, Gillet al. , 2000). Taking the potential influence from the soil microbial community on ectomycorrhizal fungi into consideration, the extinction of ectomycorrhizal fungi is a potential sign of soil microbial community degradation, thereby linking the degradation of the soil microbial community to other ecosystem components.

In order to elucidate the relationship of the extinction of ectomycorrhizal fungi species with the degradation of a soil microbial community, we chose Hengduan mountain as the sampling site. It is located in the eastern edge of Tibet plateau and is a hotspot of global biodiversity. Several types ectomycorrhizal fungi species, especially T.matsutake, have shown signs of regional extinction due to climatic and anthropogenic stresses (Ruth et al. , 2008, Yinet al. , 2020). We sampled the top-soil along a T.matsutakeextinction gradient and investigated the variation in the associated microbial communities.

\section{Materials and Methods}

\section{Sample collection and basic soil physico-chemical properties}

Soil samples were collected from the Baima Snow Mountain National Park in Yunnan Province, near Nanren village $\left(99^{\circ} 6^{\prime} 40.65^{\prime \prime} \mathrm{E}, 28^{\circ} 34^{\prime} 59.06^{\prime \prime} \mathrm{N}\right.$, at 3,534 m). The soils were sampled from an old alpine forest dominated by Cyclobalanopsis glauca (Thunb.) Oerst on August 9, 2019 (Fig. S1A). With help from native experienced mushroom collectors, soils beneath T. matsutake were collected and considered as undegraded areas (UD). Degrading areas (DI) were classified as location where T. matsutake that did not fruit that year, and degraded areas (DD) were where no T. matsutake growth had been observed for over three years. Over three sites were selected for each soil type and divided into 10 subsamples around the fruit, marked as 1 to 10, and each subsample was about $10 \mathrm{~cm}$ away from the fruit (Fig. S1B). Each subsample was collected from a depth of 0-20 cm. For each soil type, the subsamples from each site of the same mark were mixed to form 10 samples (Zhou et al. , 2016). Using this methodology, we were able to minimize the influences and errors caused by heterogeneity and focus primarily on the state of the microbial community under the same degree of T. matsutake extinction.

Soil $\mathrm{pH}$ was measured using a $\mathrm{pH}$ meter (FE20-FiveEasyTM pH, MettlerToledo, Germany) after shaking a soil water $(1: 5 \mathrm{w} / \mathrm{v})$ suspension for $30 \mathrm{~min}$. Soil moisture was measured gravimetrically. Total organic carbon (TOC) and total nitrogen (TN) contents were measured using an elemental analyzer (VarioMAX, Elementar, Germany). Ammonium $\left(\mathrm{NH}_{4}{ }^{+}-\mathrm{N}\right)$ and nitrate $\left(\mathrm{NO}_{3}{ }^{-} \mathrm{N}\right)$ were extracted at a ratio of $10 \mathrm{~g}$ fresh soil to $100 \mathrm{~mL}$ $2 \mathrm{M} \mathrm{KCl}$. After shaking for $1 \mathrm{~h}, \mathrm{NH}_{4}{ }^{+}-\mathrm{N}, \mathrm{NO}_{3}{ }^{-}-\mathrm{N}$ contents in the filtered extracts were analyzed using a continuous flow analytical system (San ++ System, Skalar, Holland). Total phosphorus content (TP) was measured using the alkali fusion-Mo-Sb Anti spectrophotometric method.

\section{DNA extraction and amplicon sequencing}

Total DNA was extracted according to the protocol for the FastDNA ${ }^{\mathrm{TM}}$ SPIN kit (MP Biomedicals), followed by measuring the DNA concentrations and quality using a Nano-100 NanoDrop spectrophotometer. Primers for the 16S rRNA gene amplification targeted the V3-V4 hypervariable region and included 338F 5'ACTCCTACGGGAGGCAGCA-3' and 806R 5'-GGACTACHVGGGTWTCTAAT-3'. The primers for ITS region amplification, 3F 5'-GCATCGATGAAGAACGCAGC-3' and 4R 5'-TCCTCCGCTTATTGATATGC3', targeted the ITS2 genetic region. PCR products were mixed in equimolar ratios according to the GeneTools Analysis Software (Version 4.03.05.0, SynGene), and the pooled PCR products were purified using an E.Z.N.A. Gel Extraction Kit (Omega, USA). Sequencing libraries were generated using the NEBNext(r) 
Ultra II DNA Library Prep Kit for Illumina(r) (New England Biolabs, MA, USA) following manufacturer's recommendations, and indexing adapters were added. Library quality was assessed with a Qubit@ 2.0 fluorometer (Thermo Fisher Scientific, MA, USA). The library was then sequenced on an Illumina Nova6000 platform to generate 250 bp paired-end reads (Guangdong Magigene Biotechnology Co., Ltd., Guangzhou, China).

The raw sequencing data generated in this study are available in the NCBI Sequence Read Archive under the accessions PRJNA687558 (bacterial undegraded stage-BUD library), PRJNA690166 (bacterial degrading stage-BDI library), PRJNA690139 (bacterial degraded stage-BDD library), PRJNA690207 (fungal undegraded stage-FUD library), PRJNA690181 (fungal degrading stage-FDI library), and PRJNA690169 (fungal degraded stage-FDD library).

Sequence data preprocessing and bioinformatics analyses

Fastp (version 0.14.1) was used for quality control of the raw sequence data using a sliding window (-W4M20). Primers were also removed using the cutadapt software program. Paired-end clean reads were then merged using the usearch -fastq_mergepairs command based on overlap between paired-end reads. Merging criteria included requiring at least $16 \mathrm{bp}$ of overlap from reads generated from the opposite end of the same DNA fragment with a maximum of 5 mismatches being allowed in the overlap region. The merged sequences were identified as raw sequences. Fastp (version 0.14.1) was then used again to perform quality control on the raw sequence data using a sliding window (-W4-M20) to obtain paired-end clean sequences. Sequences with more than $97 \%$ nucleotide similarity were clustered into operational taxonomic units (OTUs).

\section{Bioinformatic analyses}

Network analyses were conducted according to the molecular ecological network analysis pipeline (MENA, http://ieg2.ou.edu/MENA/) protocols. A cutoff threshold of 0.88-0.89 was used, and threshold values ranging from 0.01 to 0.99 over 0.01 intervals were applied to the Spearman correlation matrix (Deng \& Zhou, 2015). Module hubs and connectors were determined based on the criteria of $\mathrm{Zi}>2.5$ and $\mathrm{Pi}>0.625$, respectively. The constructed network and sub-networks were visualized using Cytoscape 3.3.0 (Xie et al. , 2020). Statistically significant differences in basic soil properties and diversity indices among the three soil types were measured using one-way ANOVA tests. In addition, differences in the relative abundances of OTUs and functional gene modules were evaluated using the Kruskal-Wallis method. Calculations of richness, Shannon index, and equitability, in addition to Linear discriminant analysis Effect Size (LEfSe) tests, principal coordinates analysis (PCoA) based on Bray-Curtis distances, and 16S rRNA gene-based functional predictions (by comparison to the database COG-cluster of orthologous groups), were all conducted with the online platform http://www.magichand.online/h5-BioCloud-site/\#/. Factor extractions were conducted using a correlation matrix in the SPSS software suite.

\section{Results}

Variation in basic soil abiotic and biotic properties during degradation

The degradation of T. matsutake habitat was accompanied by significant variation in soil physico-chemical and microbial community properties. The contents of TC, TN, TP, ammonia, nitrate, and pH significantly increased with degradation status (Table S1). The most prominent increase was for ammonia content that was nearly 42 times higher in DD soils than in UD soils, while TC, TN, and TP increased by only approximately 1.0-, 2.0-, and 0.5-fold, respectively. TN and nitrate contents did not significantly differ between UD and DI soils. The Shannon indices of the bacterial and fungal communities from UD soils were 2.28 and 0.97 , respectively, but increased to 2.77 and 1.60 in DI soils. The Shannon index values were lowest in DD soils, at 2.03 and 0.72 for bacterial and fungal communities, respectively. Similar trends were also observed for richness and equitability metrics (Fig. S2). Variations in the relative abundances (RA) of fungal and bacterial populations were observed among communities (Fig. S3A and B). The RA of $\alpha$-Proteobacteria (the bacterial phylum with the highest RA) increased from $21.9 \%$ (UD) to $31.6 \%$ (DI), and then decreased to $13.6 \%$ (DD) in the respective soil communities. Acidobacteria RAs significantly increased to as high as 
$62.7 \%$ in DD soils. The RAs of $\beta$-Proteobacteria decreased from $17.7 \%$ in UD soils to $1.1 \%$ in DD soils, while the RAs of Actinobacteria also decreased from $16.9 \%$ in UD soils to $4.4 \%$ in DD soils.

The RAs of "other" fungi decreased from $28.1 \%$ in UD soils to $6.7 \%$ in DD soils. Furthermore, the RAs of Eurotiales ,Thelephorales, and Hypocreales exhibited similar declining trends. Helotiales RAs slightly increased from $4.2 \%$ in UD soils to $7.5 \%$ in DD soils. The dominant Agaricales (41.5\% RA) of UD soils was replaced by Atheliales (73.3\% RA in DD soils).Sebacinales and Russulales exhibited the highest RAs in DI soils (18.8\% and $33.6 \%$, respectively) which were significantly higher $(p<0.05)$ than in UD and DD soils. The RA of T.matsutake in the fungal community decreased from $41 \%$ in UD soils to $1.6 \%$ in DD soils, highlighting the decreasing dominance of T.matsutake during the degradation process (Fig. S3C). LEfSe analysis indicated that T.matsutake was the most enriched representative species in UD soils, with decreasing prominence in degraded soils (Fig. S3D). Only the RAs of Piloderma olivaceumwere significantly enriched in DD soils compared to the other two degradation gradients.

The microbial community in DD exhibited a greater number of predicted gene modules related to stress resistance and basic metabolism than in DI and UD, but also exhibited fewer predicted modules related to interaction, reproduction, and cofactor metabolism. In the T. matsutake degraded habitats, the RAs of gene modules related to basic metabolites (i.e., carbohydrates, lipids, and amino acids) were enhanced, while carbohydrate metabolite-related gene modules exhibited the largest increased abundances, increasing to approximately $25 \%$ higher in the DD soils compared to UD soils. The predicted RAs of defense and motility related gene modules were also higher in DD (Fig. 1). In contrast, the predicted RAs of replication-related gene modules decreased from UD to DD, the largest decrement was chromatin structure and dynamicsrelated gene modules, with the largest decreases associated with chromatin structure and dynamics-related gene modules the RA in DD soils were only $77.5 \%$ of levels in UD soils. The RAs of community interaction and cofactor metabolite-related gene modules were also predicted as lower in DD (Fig.1).

\section{Microbial community network variation during degradation and interactions with T.matsutake}

Microbial networks become less interactive along the T.matsutake degradation gradient, although the largest network was observed for DI communities. The network topological parameters (Table S2) demonstrated that DI soil networks exhibited the most nodes $(1,280)$ and interactions $(1,414)$, although it is not the most interactive network among the three soil types. Across the degradation gradient, the average degree (avgK) decreased from 3.54 (UD) to 1.63 (DD), indicating a lower interaction density (Deng \& Zhou, 2015). Furthermore, the average clustering coefficient (avgCC) decreased from 0.192 (UD) to 0.112 (DD), while the centralization of eigenvector centrality (CE) increased from 0.231 (UD) to 0.572 (DD). These observations indicate that the size proportion of unit modules in the network decreased across the degradation gradient, with an increase in the number of smaller modules (Deng \& Zhou, 2015). The transitivity (trans) decreased from 0.506 (UD) to 0.241 (DD) across the gradient, reflecting a potentially decreased ability to communicate and cooperate among network members (Deng \& Zhou, 2015). In addition, a mantel test indicated the presence of significant differences between the empirical network and random networks $(p<0.05)$, suggesting that the empirical networks were reasonable.

The form and state of interactions in the network varied during degradation (Fig. 2). The UD network contained four module hubs (BOTU79, BOTU257, BOTU99 and FOTU7) and two bacterial connectors (BOTU175 and BOTU2394), while the DI network contained twelve module hubs and two fungal connectors (FOTU1608 and FOTU1160), and the DD network had no connectors and only a single module hub (BOTU37). The ratio of module hubs/connectors increased from UD to DD, indicating that the interaction types shifted from an inter-module focused state to an intra-module focused state (Fig. 2A and Fig. 2B).

The ratios of total positive to negative interactions $(\mathrm{p} / \mathrm{n})$ in the UD, DI, and DD networks were nearly equivalent, while the DI network had a slightly higher ratio of negative interactions than the UD and DD networks (Fig. 3A). However, the $\mathrm{p} / \mathrm{n}$ of bacterial-fungal interactions decreased from 1.18 (UD) to 0.82 (DD), while the $\mathrm{p} / \mathrm{n}$ of bacterial-bacterial interactions increased from 0.89 (UD) to 1.08 (DD). The $\mathrm{p} / \mathrm{n}$ of fungal-fungal interactions was lowest in the DI network (1.07) compared to 1.72 and 1.85 in the UD and 
DD networks, respectively (Fig. 3A). This indicated interactions between bacterial and fungi transitioned from a more cooperative to a more competitive state during degradation. The proportion of bacterial-fungal interactions to total interactions was similar among the three networks, but the proportion of fungal-fungal interactions decreased from $28.18 \%$ in the UD network to $24.31 \%$ in the DD network, while the proportion of bacterial-bacterial interactions increased from $26.01 \%$ in the UD network to $31.33 \%$ in the DD network (Fig. 3B). This observation indicates the potential decreasing relative importance of fungal interactions compared to bacterial interactions in the network along the degradation gradient.

Furthermore, the RAs of OTUs that were directly associated with T.matsutake encompassed 13 negatively interacting OTUs that had significantly lower RAs in the DD communities compared to the UD communities (Fig. 3C). However, half of the positively associated OTUs ( $80 \%$ were bacterial) exhibited increased RAs along the degradation gradient, indicating that they improved their fitness once they no longer had interactions with T.matsutake. Only BOTU43 and FOTU304 exhibited decreased RAs with the loss of T.matsutake dominance, indicating that they may have intimate and irreplaceable interactions with T.matsutake (Fig. 3C). Thus, the location of T.matsutakein the networks became marginalized as degradation increased (Fig. 2B), moving from a large central module in the UD network that had tight interactions, to a small peripheral module that had few interactions with other nodes and modules in the DD network. This change indicated the loss of functional interactions with T.matsutake during habitat degradation.

\section{The contribution of microbial community interactivity to T.matsutake extinction}

A strong linear relationship was observed between T.matsutake RAs and network structures, with $\mathrm{R}^{2}$ values as high as $0.909(p<0.05)$, further implicating the influence of network interactions on T.matsutake RAs (Fig. 4). However, correlations of soil properties in addition to the diversity and composition metrics for the bacterial and fungal communities exhibited weak linear relationships to T.matsutake RAs. The lowest $\mathrm{R}^{2}$ was observed for the relationship between community structure and T.matsutake RAs (0.006). Furthermore, a weak positive relationship was observed between community diversity and T.matsutake RAs $\left(\mathrm{R}^{2}=0.302\right)$, while a weak negative relationship was observed between soil properties and T. matsutake RAs $\left(\mathrm{R}^{2}=0.417\right)$.

\section{Discussion}

\subsection{The properties of soil microbial community degradation}

In this study, microbial community degradation was accompanied by significant variation in community composition, $\alpha$-diversity and functions. The $\alpha$-diversity rose and then decreased again, which indicated the potential error to describe microbial community degradation by $\alpha$-diversity as a proxy (Fig. S2). Degradation is triggered by stresses ( $\mathrm{Li}$ et al. , 2013), which alters soil properties in a spatially and temporally heterogeneous manner (Pedersen et al. , 2015, Jianet al. , 2018, Li et al. , 2020), creating new niches that could harbor new species (Seaton et al. , 2020). However, with increasing degradation, soil properties are more likely to be altered to an extent that the number of niches decreases, filtering species (Orrock, 2020). Thus, the relationship between $\alpha$-diversity and degradation varies from the extent (or stage) of degradation.

We found that during degradation, microbial functions focused more on stress resistance and basic metabolite, and less on interaction and reproduction (Fig.1). This indicated microbial community was under pressure such that its members reduced energy utilization efficiency to overcome the stressor(s) (Malik et al. , 2018). When facing acidic stresses, microbes expend extra energy to synthesis stress tolerance protein and, thus, leaving less energy for reproduction (Malik et al. , 2018). Further, our results supported that the microbial community lost metabolite cooperation during degradation as shown by the decrease in the relative abundance of gene modules related to microbial interaction and increase in the relative abundance of gene modules related to basic metabolism, which coincided with the decrease in microbial community interactivity (Fig.1, Fig.2B and Table S2). This indicate that under UD, members in the network were able to conserve energy by scaling back the synthesis enzymes and proteins for entire pathways, achieve high energy acquisition and reproduction rate through the exchange of intermediate metabolites (Damore \& Gore, 2012, Maliket al. , 2018). The broken metabolite cooperation under stress conditions forced members to express a great number of basic metabolite related gene to ensure survival (Damore \& Gore, 2012, Malik 
et al. , 2018). We found the inhibited metabolite cooperation occurred more frequently between fungi and bacteria than between fungi and fungi or bacteria and bacteria, since the positive interaction ratio of the former consistently decreased over the degradation gradient (Fig.3A). In nature, fungi play an important role in litter degradation and contribute the main driving force for organic carbon release to the soil (Taylor \& Osborn, 1996), while bacteria play a larger role in various ecosystem functions such as ammonia oxidization and nitrogen fixation. (Galloway et al. , 2004). The decreased cooperation between fungi and bacteria is a potential indicator of decoupling between their respective functions (Taylor \& Osborn, 1996, Galloway et al. , 2004, Kuypers et al. , 2018). This was further supported by the loss of inter-module interactions (Fig. 2A and Fig. 2B), since members within the same module are more likely to be linked to a single function, decreasing inter-module interaction indicates decoupling of different functions (Deng \& Zhou, 2015). As discussed above, our result support that the interactivity, rather than simple diversity, is better able to indicate microbial community degradation. Due to the extremely high richness and functional redundancy of the microbial community, it is more meaningful to understand who's working or interacting together rather than who is simple present (Curtis, 2006).

\subsection{Reduced interactivity lead to T.matsutake extinction}

Interactions among community members are an important contributor to community stability and species diversity (Mougi \& Kondoh, 2012, Haleet al. , 2020). The interactions could be cooperation and competition in metabolism, immune, stress tolerance, and appeared as positive or negative interactions (Mougi \& Kondoh, 2012).

As interactivity within the soil microbial community decreased during degradation, T.matsutakelost cooperative interactions (Fig.3C) that may have been capable of enhancing the rates of certain ecological processes (Corbett \& Watkin, 2018, Cui et al. , 2019), stimulating energy conservation by individual members (Malik et al. , 2018), and even allowing the coexistence of certain species (Cai et al. , 2020). The loss of cooperation increases the cost of survival for that species. We found that T.matsutake was marginalized from a central module to a peripheral one within the networks (Fig.2B). As a module is a collection of members (nodes) that are potentially highly functionally related (Deng \& Zhou, 2015), this indicated that T.matsutake could not rely on the original functional groups for metabolic cooperation, and resulted in extinction. (Gill et al. , 2000, Xu et al. , 2015). Thus, our result support the hypothesis that loss of cooperation due to reduced interactivity of the microbial community was responsible for the extinction of T.matsutake (Gill et al. , 2000, Xuet al. , 2015). This was further confirmed by regression analysis that showed the extent of T.matsutake extinction was most linearly correlated to network interaction indicators than to soil properties, community composition or biodiversity (Fig.4).

However, our study found that all of the 13 OTUs that competed with T.matsutake had also decreased in their relative abundance during degradation, which indicated that groups with functions similar toT.matsutake were also facing the threat of extinction due to environmental stress. Competition also contributes to community stability as it is involved in resource partitioning and functional redundancy among community members (Pianka, 1981, Pinsky, 2019). The community could retain functional stability by trade-offs between the functions of several phylogenetic groups under stress (Pianka, 1981, Pinsky, 2019). Thus, it became an open question as to whether the community possessed any other groups that could functionally replace T.matsutake and its competitors. As the ectomycorrhizal species of the keystone plant (Gill et al. , 2000), T.matsutake and its competitors were helpful for maintaining the dominance of the host plant by assisting in nutrient uptake and defense against stress and disease (Liang et al. , 2020). Over the long term, the extinction of T.matsutake and its competitors are a potential threat to keystone vegetation and even other animals that inhabit the local environment (Gill et al. , 2000, Weisskopf et al. , 2021).

In summary, the microbial community, during the degradation of T.matsutake, was demostrated variation in composition and diversity, and a switch from an interactive state where metabolite and signal functions predominated to stress tolerance state. The decreased interactivity marginalized T.matsutake from a large center module to a peripheral module and reduced its interactions, leading to its extinction. The extinction caused by the degradation of microbial community directly impacts the ecosystem and is a potential long term 
threat to aboveground biodiversity through rhizosphere activity and the food-web. This study highlights that the key characteristic of microbial community degradation and the mechanism for microbial species extinction during degradation is the reduced interactivity. For future studies focusing on conservation and on microbial community, an understanding of the relationships among stress, microbial community properties, and the extent of the microbial community degradation, will allow for improved comprehensive conservation measures that target both below- and above-ground communities.

\section{Acknowledgements}

This work was supported by the Second Tibetan Plateau Scientific Expedition and Research Program (2019QZKK0402, 2019QZKK0307); the National Key Research and Development Program of China (2016YFC0502104, 2018YFA0901200); and the National Natural Science Foundation of China (41473079, 41673082, 41671270).

\section{References}

Berendsen RL, Pieterse CMJ \& Bakker PAHM (2012) The rhizosphere microbiome and plant health. Trends in Plant Science 17 : 478-486. https://doi.org/10.1016/j.tplants.2012.04.001

Bowen GD \& Theodorou C (1979) Interactions between bacteria and ectomycorrhizal fungi. Soil Biology and Biochemistry 11 : 119-126. https://doi.org/10.1016/0038-0717(79)90087-7

Cai W, Snyder J, Hastings A \& D'Souza RM (2020) Mutualistic networks emerging from adaptive niche-based interactions. Nature Communications 11 : 5470. https://doi.org/10.1038/s41467-020-19154-5

Cheng C, Gao M, Zhang Y, Long M, Wu Y \& Li X (2021) Effects of disturbance to moss biocrusts on soil nutrients, enzyme activities, and microbial communities in degraded karst landscapes in southwest China.Soil Biology and Biochemistry 152 : 108065. https://doi.org/10.1016/j.soilbio.2020.108065

Collado E, Bonet JA, Camarero JJ, Egli S, Peter M, Salo K, Martinez-Pena F, Ohenoja E, MartinPinto P \& Primicia I (2019) Mushroom productivity trends in relation to tree growth and climate across different European forest biomes. The Science of the Total Environment 689 : 602-615. https://doi.org/10.1016/j.scitotenv.2019.06.471

Corbett M \& Watkin E (2018) Microbial cooperation improves bioleaching recovery rates. Microbiology Australia 39 :50-52. https://doi.org/10.1071/MA18013

Cui J, Mai G, Wang Z, Liu Q, Zhou Y, Ma Y \& Liu C (2019) Metagenomic insights into a celluloserich niche reveal microbial cooperation in cellulose degradation. Frontiers in microbiology 10 : 618. https://doi.org/10.3389/fmicb.2019.00618

Curtis T (2006) Microbial ecologists: it's time to 'go large'.Nature Reviews Microbiology 4 : 488-488. https://doi.org/10.1038/nrmicro1455

Damore JA \& Gore J (2012) Understanding microbial cooperation. Journal of Theoretical Biology 299 : 31-41. https://doi.org/10.1016/j.jtbi.2011.03.008

De VFT \& Ashley S (2013) Controls on soil microbial community stability under climate change. Frontiers in microbiology 4 :1-16. https://doi.org/10.3389/fmicb.2013.00265

Deng Y \& Zhou J (2015) Molecular ecological network of microbial communities. Encyclopedia of Metagenomics: Genes, Genomes and Metagenomes: Basics, Methods, Databases and Tools, (Nelson KE, ed.) p.`pp. 504-510. Springer US, Boston, MA.

Firestone MPWK (2006) Response of Microbial Community Composition and Function to Soil Climate Change. Microbial Ecology 52 : 716-724. https://doi.org/10.1007/s00248-006-9103-3

Galloway JN, Dentener FJ, Capone DG, Boyer EW, Howarth RW, Seitzinger SP, Asner GP, Cleveland CC, Green PA \& Holland EA (2004) Nitrogen Cycles: Past, Present, and Future. Royal Horticultural Society. 
Gill, WM, Guerin-Laguette, Lapeyrie \& Suzuki (2000) Matsutake - morphological evidence of ectomycorrhiza formation between Tricholoma matsutake and host roots in a pure Pinus densiflora forest stand. $N E W$ PHYTOL 147 :381 - 388. https://doi.org/10.1046/j.1469-8137.2000.00707.x

Hale KRS, Valdovinos FS \& Martinez ND (2020) Mutualism increases diversity, stability, and function of multiplex networks that integrate pollinators into food webs. Nature Communications 11 : 2182. https://doi.org/10.1038/s41467-020-15688-w

Hall EK, Bernhardt ES, Bier RL, et al. (2018) Understanding how microbiomes influence the systems they inhabit. Nature Microbiology 3 : 977-982. https://doi.org/10.1038/s41564-018-0201-z

Hoeksema JD, Bever JD, Chakraborty S, et al. (2018) Evolutionary history of plant hosts and fungal symbionts predicts the strength of mycorrhizal mutualism. Communications Biology 1 : 116. https://doi.org/10.1038/s42003-018-0120-9

Jansson JK \& Hofmockel KS (2020) Soil microbiomes and climate change.Nature Reviews Microbiology 18 : 35-46. https://doi.org/10.1038/s41579-019-0265-7

Jian J, Steele MK, Day SD, Quinn Thomas R \& Hodges SC (2018) Measurement strategies to account for soil respiration temporal heterogeneity across diverse regions. Soil Biology and Biochemistry 125 : 167-177. https://doi.org/10.1016/j.soilbio.2018.07.003

Konopka A (2006) Microbial ecology: searching for principles. Microbe 1 : 175-179.

Kuypers MMM, Marchant HK \& Kartal B (2018) The microbial nitrogen-cycling network. Nature Reviews Microbiology 16 : 263-276. https://doi.org/10.1038/nrmicro.2018.9

Li J, Pei J, Pendall E, Fang C \& Nie M (2020) Spatial heterogeneity of temperature sensitivity of soil respiration: A global analysis of field observations. Soil Biology and Biochemistry 141 : 107675. https://doi.org/10.1016/j.soilbio.2019.107675

Li XL, Gao J, Brierley G, Qiao YM, Zhang J \& Yang YW (2013) Rangeland degradation on the Qinghai-Tibet plateua: implications for rehabilitation. Land Degradation \& Development 24 : 72-80. https://doi.org/10.1002/ldr.1108

Liang M, Johnson D, Burslem DFRP, Yu S, Fang M, Taylor JD, Taylor AFS, Helgason T \& Liu X (2020) Soil fungal networks maintain local dominance of ectomycorrhizal trees. Nature Communications 11 : 2636. https://doi.org/10.1038/s41467-020-16507-y

Malik AA, Jeremy P, Buckeridge KM, Tim G, Nico J, Somak C, Soon GH, Peyton JM, Mason KE \& Maaike VA (2018) Land use driven change in soil pH affects microbial carbon cycling processes. Nature Communications 9 : 3591. https://doi.org/10.1038/s41467-018-05980-1

Martin F, Kohler A, Murat C, Veneault-Fourrey C \& Hibbett DS (2016) Unearthing the roots of ectomycorrhizal symbioses. Nature Reviews Microbiology 14 : 760-773. https://doi.org/10.1038/nrmicro.2016.149

Moreno-Mateos D, Alberdi A, Morriën E, van der Putten WH, Rodríguez-Uña A \& Montoya D (2020) The long-term restoration of ecosystem complexity.Nature Ecology \&3 Evolution 4 : 676-685. https://doi.org/10.1038/s41559-020-1154-1

Mougi A \& Kondoh M (2012) Diversity of interaction types and ecological community stability. Science 337 : 349. https://doi.org/10.1126/science. 1220529

Ohara H \& Hamada M (1967) Disappearance of bacteria from the zone of active mycorrhizas in Tricholoma matsutake (S. Ito et Imai) Singer. Nature 213 : 528-529. https://doi.org/10.1038/213528a0

Orrock JL (2020) Deterministic insights from stochastic interactions.Proceedings of the National Academy of Sciences $117: 6965$. https://doi.org/10.1073/pnas.2001848117 
Pedersen LL, Smets BF \& Dechesne A (2015) Measuring biogeochemical heterogeneity at the micro scale in soils and sediments. Soil Biology and Biochemistry 90 : 122-138. https://doi.org/ 10.1016/j.soilbio.2015.08.003

Pianka E (1981) Competition and niche theory. Vol. 8 p. ^pp. 167-196.

Pinsky ML (2019) Species coexistence through competition and rapid evolution. Proceedings of the National Academy of Sciences 116 : 2407. https://doi.org/10.1073/pnas.1822091116

Ruth S, Renee M, Li H, Fang Z \& Wang Y (2008) Spatial patterns of plant diversity and communities in Alpine ecosystems of the Hengduan Mountains, Northwest Yunnan, China. Journal of Plant Ecology 2 :117-136. https://doi.org/10.1093/jpe/rtn012

Seaton FM, George PBL, Lebron I, Jones DL, Creer S \& Robinson DA (2020) Soil textural heterogeneity impacts bacterial but not fungal diversity.Soil Biology and Biochemistry 144 : 107766. https://doi.org/10.1016/j.soilbio.2020.107766

Singh BK, Bardgett RD, Smith P \& Reay DS (2010) Microorganisms and climate change: terrestrial feedbacks and mitigation options. Nature Reviews Microbiology 8 : 779-790. https://doi.org/10.1262/jrd.17004

Taylor TN \& Osborn JM (1996) The importance of fungi in shaping the paleoecosystem. Review of Palaeobotany and Palynology90 : 249-262. https://doi.org/10.1016/0034-6667(95)00086-0

Weisskopf L, Schulz S \& Garbeva P (2021) Microbial volatile organic compounds in intra-kingdom and inter-kingdom interactions. Nature Reviews Microbiology . https://doi.org/10.1038/s41579-020-00508-1

Xie F, Ma A, Zhou H, Liang Y, Yin J, Ma K, Zhuang X \& Zhuang G (2020) Niche differentiation of denitrifying anaerobic methane oxidizing bacteria and archaea leads to effective methane filtration in a Tibetan alpine wetland. Environment International 140 : 105764. https://doi.org/10.1016/j.envint.2020.105764

Xu C, Xiaofei L, Deping LV \& Mingying G (2015) Climate niche characteristics and potential distribution of Tricholoma matsutake in China. Acta Edulis Fungi .

Yin L, Dai E, Zheng D, Wang Y \& Tong M (2020) What drives the vegetation dynamics in the Hengduan Mountain region, southwest China: Climate change or human activity? Ecological Indicators 112 : 106013. https://doi.org/10.1016/j.ecolind.2019.106013

Yun Wang, Fuqiang Yu, Chunxiang Zhang \& Shuhong Li (2017) Tricholoma matsutake : an edible mycorrhizal mushroom of high socioeconomic relevance in China. Revista mexicana de micología46 : 55-61.

Zhang B, Deng H, Wang H-l, Yin R, Hallett PD, Griffiths BS \& Daniell TJ (2010) Does microbial habitat or community structure drive the functional stability of microbes to stresses following re-vegetation of a severely degraded soil? Soil Biology and Biochemistry42 : 850-859. https://doi.org/10.1016/j.soilbio.2010.02.004

Zhou J, Deng Y, Shen L, et al. (2016) Temperature mediates continental-scale diversity of microbes in forest soils. Nature communications 7 : 12083.https://doi.org/10.1038/ncomms12083

\section{Figure Caption}

Fig. 1 . The relative abundances of gene modules. The letters $a, b$, and c indicate statistically significant differences between groups $(\mathrm{p}<0.05)$. Functional modules correspond to the following designations: A-energy production and conversion, B-amino acid transport and metabolism, C-nucleotide transport and metabolism, D-carbohydrate transport and metabolism, E-intracellular trafficking, secretion, and vesicular transport, F-lipid transport and metabolism, G-transcription, H-cell wall or membrane or envelope biogenesis, I-extracellular structures, J-signal transduction mechanisms, K-coenzyme transport and metabolism, L-inorganic ion transport and metabolism, M-RNA processing and modification, N-secondary metabolite biosynthesis, transport, and catabolism, O-cell motility, P-defense mechanisms, Q-general function prediction only, R-function unknown, S-cell cycle control, cell division, chromosome partitioning, T-post-translational 
modification, protein turnover, and chaperones, U-replication, recombination and repair, V-chromatin structure and dynamics, and W-translation, ribosomal structure, and biogenesis. Gene modules with very low RAs were amplified 10-1000 times to better visualize their relative ratios across groups ( $\mathrm{M}$, I and V). The modules can be characterized into six general groups including basic metabolism-related (dark yellow), interactionrelated (gray), cofactor metabolite-related (yellow), environmental resistance-related (brown), other (blue), and reproduction-related (green) functions.

Fig. 2 Important nodes and modules within the soil microbial networks. Fig.2A shows the identity of the module hubs and connectors for the three soil types. The nodes with $\mathrm{Zi}>2.5$ were considered module hubs, and nodes with $\mathrm{Pi}>0.625$ were considered connectors, while nodes with both $\mathrm{Zi}>2.5$ and $\mathrm{Pi}>0.625$ were considered network hubs. The module hub and connector node identities at the phylum level are shown on the right side of the panels. Fig.2B shows the modularity of soil networks and the location of T.matsutake within the networks. Blue circles show T.matsutake, yellow circles show bacterial OTUs, and green circles show fungal OTUs. Yellow and green lines show positive and negative interactions, respectively. Several smaller modules that were highly marginalized are not included in the figure to allow for better visualization of each stage's network.

Fig. 3 . The interactions and interactive OTUs associated with T.matsutake. Fig.3A shows the ratio of positive to negative interactions. Fig.3B shows the relative abundances of different interaction types across the T.matsutake habitat degradation gradient. + and - indicate positive and negative interactions, respectively. B and $\mathrm{F}$ indicate bacterial and fungal nodes, respectively, wherein $\mathrm{B}+\mathrm{F}$ indicates inferred positive interactions between bacterial OTUs and fungal OTUs. Fig.3C shows variation in the relative abundances of OTUs that originally interacted with T.matsutake, ${ }^{* * *}$ indicates statistically significant differences among groups $(\mathrm{p}<$ $0.001)$.

Fig. 4. The linear relationships between T.matsutake RAs and soil properties, community diversity, community structure, and community network structures. Upward arrows indicate components that were positively related to extraction. BE, BR, and BS indicate bacterial equitability, richness, and Shannon index values, respectively, while FE, FR, and FS indicate the respective fungal counterparts. Community structure was extracted from $\mathrm{PC} 1$ of the bacterial and fungal PCoAs, while network structure was comprised of the values from the network indices avgK, avgCC, and Tran. Shaded areas show 95\% confidence intervals.

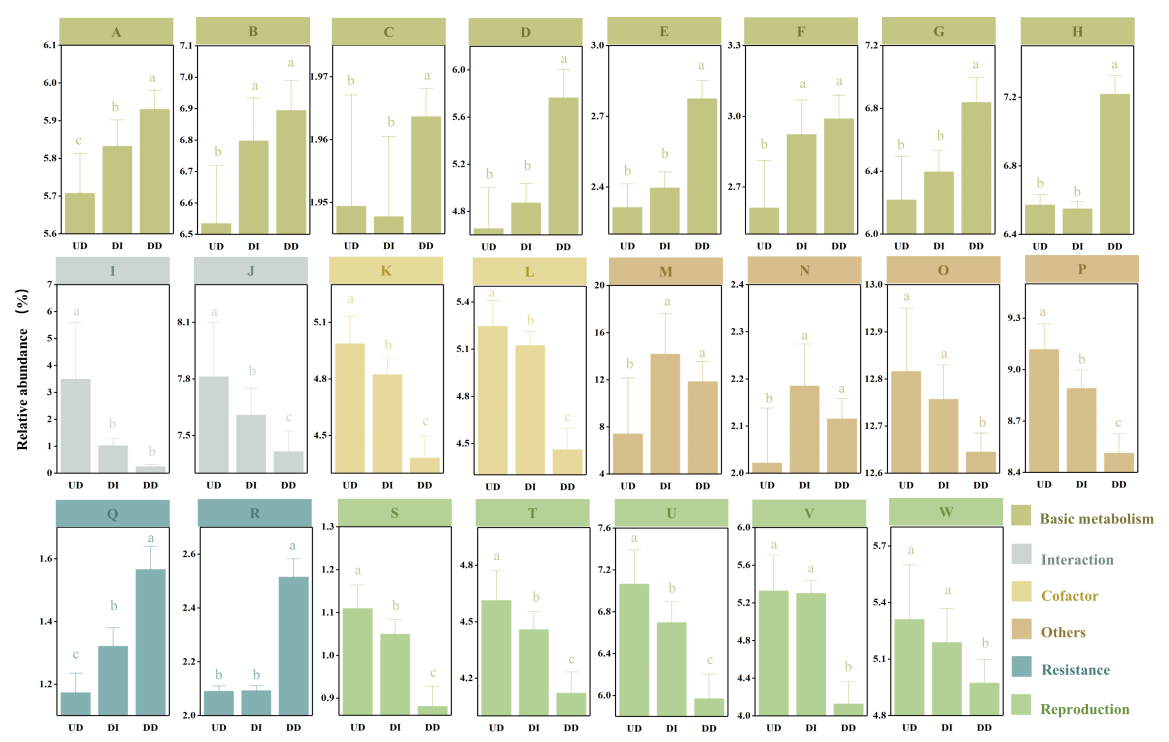




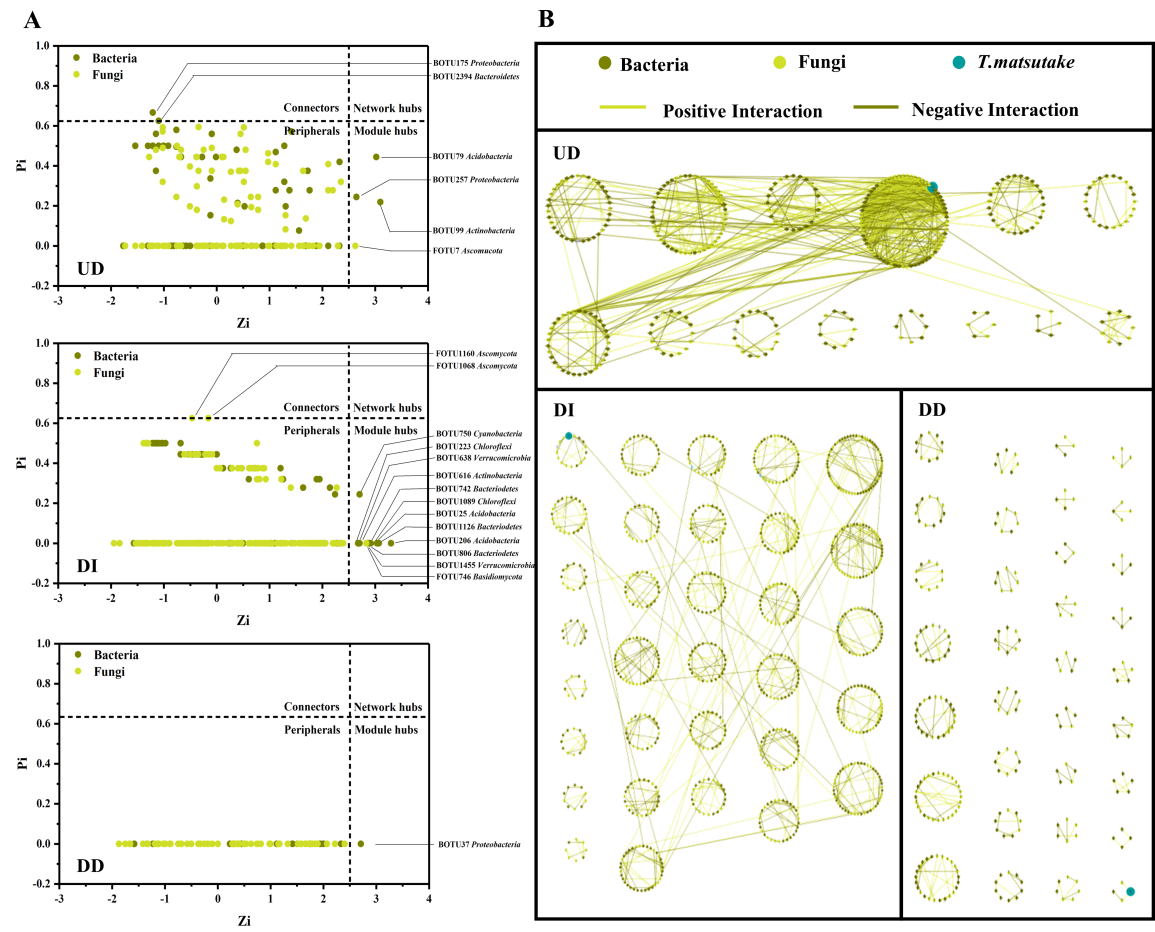




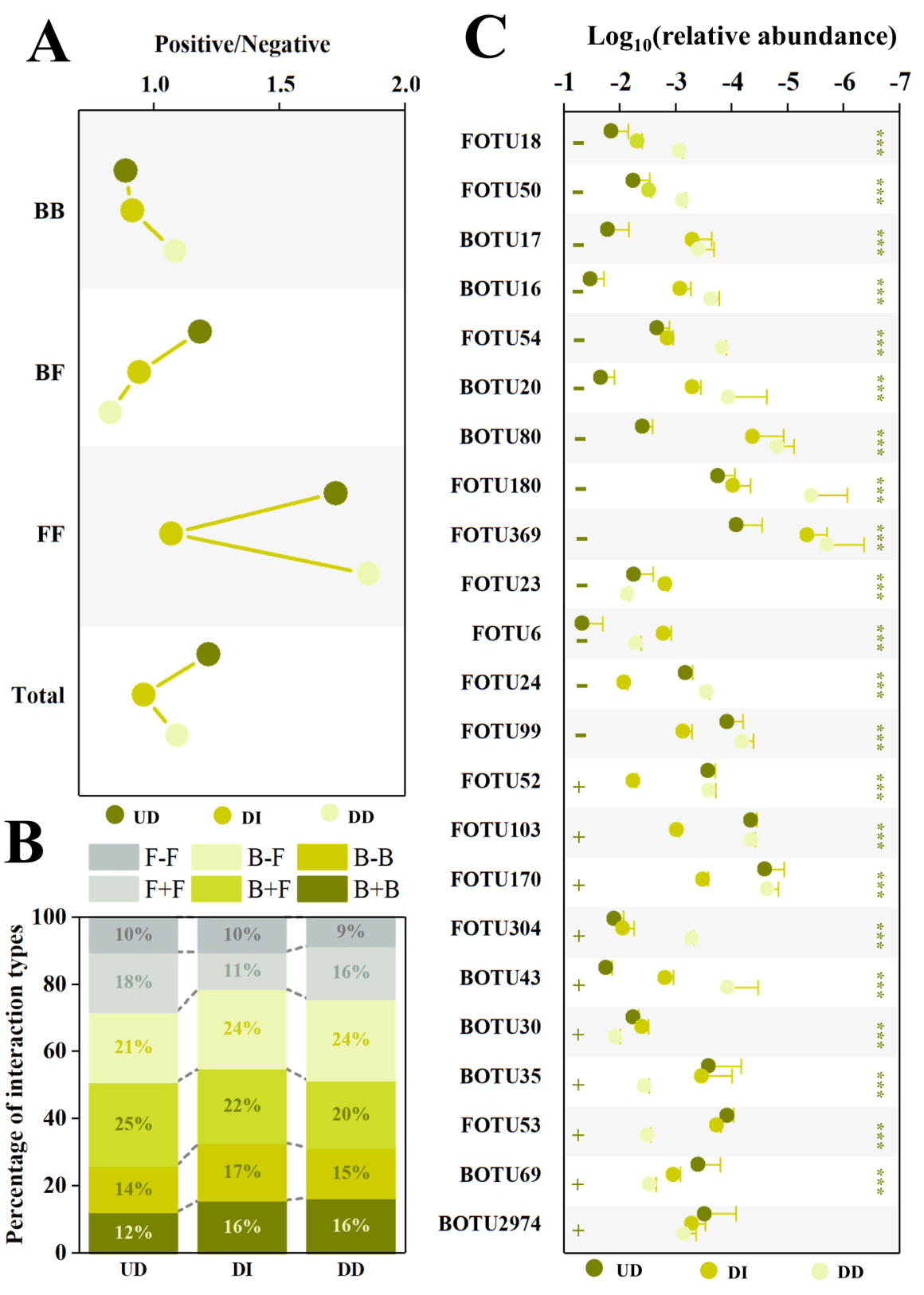



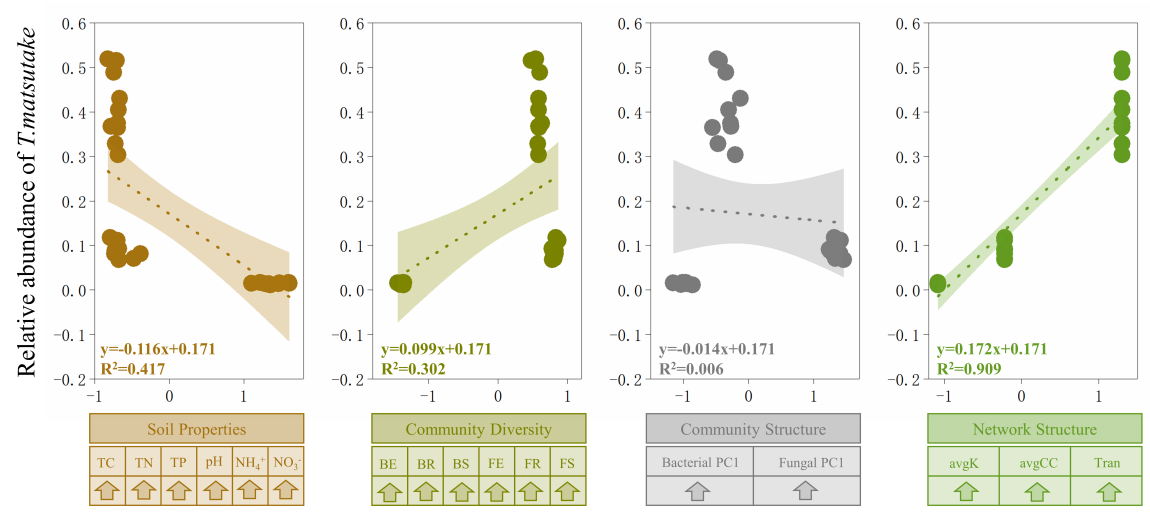